LA GRANJA:

REVISTA DE

CIENCIAS DE LA VIDA

pISSN:1390-3799; eISSN:1390-8596

http:/ / doi.org/10.17163/lgr.n31.2020.07
Artículo científico / Scientific paper

CIENCIAS VETERINARIAS

\title{
META-ANÁLISIS DEL EFECTO DE LA INCLUSIÓN ALIMENTICIA DE GLUTAMINA SOBRE EL DESEMPEÑO PRODUCTIVO EN LECHONES
}

\author{
META-ANALYSIS OF THE EFFECT OF GLUTAMINE DIETARY INCLUSION ON \\ PRODUCTIVE PERFORMANCE IN PIGLETS
}

Jimmy Rolando Quisirumbay Gaibor (i)

Facultad de Medicina Veterinaria y Zootecnia. Universidad Central del Ecuador, Av. Universitaria, Quito, 170129, Ecuador.

*Autor para correspondencia: jrquisirumbay@uce.edu.ec

Manuscrito recibido el 21 de abril de 2019. Aceptado, tras revisión, el 24 de enero de 2020. Publicado el 1 de marzo de 2020.

\begin{abstract}
Resumen
La glutamina no es considerada un aminoácido esencial; sin embargo, juega un rol importante en la salud y crecimiento de neonatos y adultos. En lechones el destete genera atrofia de las vellosidades intestinales y retraso en el crecimiento. Varios trabajos han demostrado que la suplementación de glutamina (0,2-2\%) disminuye los efectos adversos del estrés post-destete en lechones. El objetivo de este manuscrito fue evaluar el tamaño de efecto de la suplementación de glutamina sobre el rendimiento productivo de lechones, la consistencia de su efecto y la influencia de otros factores mediante el uso de meta-análisis. La administración de glutamina mejora la conversión alimenticia $(p<0,001)$, y los lechones que reciben glutamina convierten mejor el alimento cuando la suplementación duró entre 7 a 14 días $(p=0,0023)$, pues requieren 121,6 $\mathrm{g}$ menos de alimento en comparación con el grupo control para hacer $1 \mathrm{~kg}$ de peso vivo. Cuando la suplementación se realiza por un periodo de 15 a 30 días y 7 a 30 días, el ahorro de alimento es de 70,6 $g(p<0,001)$ y 87,3 $g(p<0,001)$ por $\mathrm{kg}$ de peso vivo respectivamente. La ganancia diaria de peso es superior en 20,3 g/día ( $p=0,0029)$ frente al grupo control entre los 7 a 30 días de suplementación y de 28,2 g/día $(p=0,0002)$ entre los 15 a 30 días. La edad y peso del lechón al inicio de la suplementación, el nivel de lisina, la proteína cruda y el número de repeticiones por tratamiento influyen en el efecto de la glutamina sobre las variables evaluadas.
\end{abstract}

Palabras clave: nutrición, dieta, nutrientes, aminoácidos, cerdos. 


\begin{abstract}
Glutamine is not considered an essential amino acid; however, it plays an important role in the health and growth of neonates and adults. In piglets, weaning generates atrophy of the intestinal villi and growth retardation. Several studies have shown that glutamine supplementation (0.2-2\%) decreases the adverse effects of post-weaning stress in piglets. The aim of this article was to evaluate the effect size of glutamine supplementation on the productive performance of piglets, the consistency of their effect and the influence of other factors through the use of metaanalysis. The administration of glutamine improves the feed conversion $(p<0.001)$, the piglets that receive glutamine convert the feed better when the supplementation lasted between 7 to 14 days $(p=0.0023)$, since they require $121.6 \mathrm{~g}$ less of feed in comparison with the control group to make $1 \mathrm{~kg}$ of body weight. When the supplementation is done for a period of 15 to 30 days and 7 to 30 days, the saving of feed is $70.6 \mathrm{~g}(p<0.001)$ and $87.3 \mathrm{~g}(p<0.001)$ per $\mathrm{kg}$ of body weight, respectively. The daily weight gain is higher in $20.3 \mathrm{~g} /$ day $(p=0.0029)$ compared to the control group between 7 to 30 days of supplementation and $28.2 \mathrm{~g} /$ day $(p=0.0002)$ between 15 to 30 days. Age and weight of the piglet at the beginning of the supplementation, level of lysine, crude protein and the number of repetitions per treatment influence on the effect of glutamine on the variables evaluated.
\end{abstract}

Keywords: nutrition, diet, nutrients, amino acids, pigs.

Forma sugerida de citar: Quisirumbay Gaibor, J.R. (2020). Meta-análisis del efecto de la inclusión alimenticia de glutamina sobre el desempeño productivo en lechones. La Granja: Revista de Ciencias de la Vida. Vol. 31(1):96-107. http://doi.org/10.17163/lgr.n31.2020.07.

IDs Orcid:

Jimmy Rolando Quisirumbay Gaibor: https:/ / orcid.org/0000-0003-1612-8503 


\section{Introducción}

El destete, el estrés por transporte y el estrés térmico tienen el potencial de incrementar la incidencia de enfermedades, especialmente cuando estos ocurren simultáneamente (Varley y Wiseman, 2001). Durante el proceso de destete, el estrés puede ser inducido por la separación del lechón de la madre, la reubicación y la mezcla de camadas, adicionalmente pueden existir cambios bruscos en la dieta que reducen o eliminan el consumo de alimento en las primeras horas de vida (Wijtten, Meulen y Verstegen, 2011; Campbell, Crenshaw y Polo, 2013). El estrés durante el destete altera el desarrollo de las funciones de barrera del tracto gastro-intestinal que conduce a consecuencias perjudiciales permanentes para la salud intestinal durante la vida del cerdo (Moeser, Pohl y Rajput, 2017; Pluske, Turpin y Kim, 2018). Para combatir los efectos negativos del destete sobre la salud del lechón se han implementado varias estrategias entre las cuales se encuentra el manejo de la edad del destete, el acondicionamiento ambiental, la manipulación nutricional y el uso de antibióticos a través de la dieta (Lalles y col., 2007; Gresse y col., 2017; Solà-Oriol y Gasa, 2017). En particular, los antibióticos en la dieta pueden permitir al lechón destetado reducir la carga patógena y promover el crecimiento (Cromwell, 2002). Sin embargo, su uso está siendo limitado debido a la posible contribución de los antibióticos al desarrollo de líneas de bacterias resistentes a los antibióticos (Smith y col., 2010).

La suplementación dietaria de glutamina disminuye los problemas de salud, previene la atrofia intestinal, mantiene el estatus antioxidante, disminuye la incidencia de diarreas, lo que resulta en un incremento de la ganancia de peso y mejora de la eficiencia alimenticia (Haynes y col., 2009; Zhong y col., 2011; Wang y col., 2014; Watford, 2015). También se han reportado resultados similares en pollos de engorde (Nassiri Moghaddam y AlizadehGhamsari, 2013; Jazideh y col., 2014; Nascimento y col., 2014; Manvailer y col., 2015; Muro y col., 2015; Olubodun y col., 2015; Ribeiro Jr y col., 2015; Luquetti y col., 2016; Maiorka y col., 2016; Namroud y col., 2017). La glutamina es el combustible prefe- rido de las células intestinales y del sistema inmune (Horio y col., 2008; Sakiyama y col., 2009; Zhong y col., 2012), la suplementación de este aminoácido tiene por objetivo mantener la función intestinal a través del destete (Curi y col., 2007). Diferentes investigaciones demuestran que la glutamina es un aminoácido abundante en los fluidos fisiológicos y en las proteínas corporales y es un regulador de la expresión genética (Wu y col., 2011; Xi y col., 2011). La glutamina sirve como un sustrato energético para las células de rápida división y la construcción de polipéptidos y proteínas (Rhoads y Wu, 2009), y es un precursor esencial de moléculas bioactivas (Boza y col., 2000). La producción endógena de Gln en mamíferos es insuficiente durante condiciones adversas y existe evidencia que la suplementación de Gln puede ser necesaria en la alimentación de animales jóvenes durante estados de mal nutrición (Wu, Meier y Knabe, 1996; Chamorro y col., 2010).

Existen numerosos trabajos en los cuales se ha investigado la suplementación dietaria de glutamina con la finalidad de mejorar el desempeño productivo del lechón. Sin embargo, varios han sido los niveles de inclusión dietaria y las circunstancias experimentales bajo las cuales se han desarrollado estos estudios. El objetivo de este manuscrito fue evaluar el tamaño de efecto de la suplementación de Gln sobre el rendimiento productivo de lechones, la consistencia de su efecto y la influencia de otros factores mediante el uso de la herramienta estadística de meta-análisis.

\section{Materiales y métodos}

\subsection{Fuente de Información (datos)}

Se realizó una búsqueda electrónica de artículos científicos en revistas indexadas con revisión doble ciego en las siguientes bases electrónicas: $C A B$ direct, Elsevier biobase-CABS, Google Scholar, MEDLINE, PubMed, Science Direct (Journal), Scopus, Academic Search Complete, CAB Abstract, Directory of Open Access Journals. Se utilizó una combinación de palabras clave: glutamina, aminoácidos, dieta, alimento, nutrición, lechones, destete y sus equivalentes en inglés, sin restricciones de fecha. 
Tabla 1. Estadísticos descriptivos (covariables)

\begin{tabular}{ccccc}
\hline & Promedio & Mínimo & Máximo & Moda \\
\hline $\begin{array}{c}\text { Nivel de inclusión de Gln } \\
\text { en alimento (\%) }\end{array}$ & 0,96 & 0,20 & 2,00 & 1,00 \\
\hline $\begin{array}{c}\text { Duración de la } \\
\text { suplementación (días) }\end{array}$ & 16,34 & 7,00 & 30,00 & 14,00 \\
\hline $\begin{array}{c}\text { Peso vivo (kg) inicio de la } \\
\text { suplementación }\end{array}$ & 6,34 & 4,96 & 9,22 & 5,78 \\
\hline $\begin{array}{c}\text { Edad inicio de la } \\
\text { suplementación (kg) }\end{array}$ & 22,91 & 17,00 & 28,00 & 21,00 \\
\hline $\begin{array}{c}\text { Nivel de lisina en la dieta } \\
(\%)\end{array}$ & 1,33 & 1,17 & 1,60 & 1,30 \\
\hline $\begin{array}{c}\text { Nivel de PC en la dieta } \\
(\%)\end{array}$ & 21,32 & 18,08 & 23,74 & 21,09 \\
\hline $\begin{array}{c}\mathrm{N}^{\circ} \\
\text { repeticiones/tratamiento }\end{array}$ & 5,42 & 2,00 & 12,00 & 3,00 \\
\hline $\mathrm{N}^{\circ}$ repeticiones/control & 5,39 & 2,00 & 12,00 & 3,00 \\
\hline $\mathrm{PC}=$ proteína cruda & & & &
\end{tabular}

\subsection{Criterios de Inclusión}

Se seleccionaron aquellos artículos en los cuales se administró glutamina exclusivamente a través de la dieta y en lechones. Los artículos debían incluir información respecto al número de unidades experimentales (repeticiones) por tratamiento; los experimentos debían incluir al menos 2 tratamientos (incluyendo el grupo control: sin Gln), peso vivo y edad del lechón al inicio del periodo de estudio, ni- vel de glutamina suplementado a través del alimento y duración de la suplementación, nivel de lisina y proteína cruda en el alimento utilizado (covariables). Además, debía incluir al menos una de las variables respuesta de interés: ganancia diaria de peso (GDP), consumo diario promedio de alimento (CDPA) y conversión alimenticia (CA). Debían incluir media (promedio) y alguna medida de variación (desviación estándar (SD), error estándar (SE)).

Tabla 2. Resumen de las variables respuesta en los parámetros productivos

\begin{tabular}{cccccc}
\hline \multirow{2}{*}{ Parámetro productivo } & \multirow{3}{*}{ Meta-análisis } & \multicolumn{4}{c}{ Resumen variable respuesta } \\
\cline { 3 - 6 } & & \multicolumn{2}{c}{ Tratamiento } & \multicolumn{2}{c}{ Control } \\
\cline { 2 - 6 } & & Media & SD & Media & SD \\
\hline \multirow{3}{*}{ GDP $(\mathrm{kg} / \mathrm{di} a)$} & 7 a 30 días & 0,22 & 0,12 & 0,20 & 0,11 \\
\cline { 2 - 6 } & 7 a 14 días & 0,16 & 0,10 & 0,15 & 0,09 \\
\cline { 2 - 6 } & 15 a 30 días & 0,31 & 0,10 & 0,29 & 0,10 \\
\hline \multirow{3}{*}{ CDPA $(\mathrm{kg} /$ día $)$} & 7 a 30 días & 0,33 & 0,17 & 0,33 & 0,15 \\
\cline { 2 - 6 } & 7 a 14 días & 0,25 & 0,12 & 0,26 & 0,10 \\
\cline { 2 - 6 } & 15 a 30 días & 0,46 & 0,16 & 0,44 & 0,14 \\
\hline \multirow{3}{*}{$\mathrm{CA}(\mathrm{kg} / \mathrm{kg})$} & 7 a 30 días & 1,81 & 0,89 & 1,94 & 1,12 \\
\cline { 2 - 6 } & 7 a 14 días & 1,98 & 1,08 & 2,14 & 1,37 \\
\cline { 2 - 6 } & 15 a 30 días & 1,51 & 0,28 & 1,61 & 0,32 \\
\hline
\end{tabular}

$\mathrm{SD}=$ desviación estándar 


\subsection{Análisis Estadístico}

Se localizaron un total de 13 artículos científicos que cumplieron con los criterios de inclusión anteriormente mencionados (Wu, Meier y Knabe, 1996; Lee y col., 2003; Domeneghini y col., 2004; Zhou y col., 2006; Zou y col., 2006; Abreu y col., 2010; Hsu y col., 2010; Shan y col., 2012; Xiao y col., 2012; Wang y col., 2014; He y col., 2016; Duttlinger y col., 2019). De todos los 13 manuscritos se extrajeron los datos de las variables de interés, es importante mencionar que la técnica de meta-análisis considera a todos los artículos que cumplen los criterios de inclusión (13) y no realiza un muestreo de los mismos, pues uno de sus propósitos es tener un n (número de repeticiones) lo más grande posible. Para el procesamiento estadístico de los datos se utilizó MIX 2.0 Pro en Microsoft Excel (Bax, 2016). Se determinó el tamaño del efecto de la suplementación de glutamina por diferencia de medias (DM) entre el grupo tratamiento y el control, con intervalos de confianza al $95 \%$. La heterogeneidad se evaluó por medio del índice de inconsistencia $\left(I^{2}\right)$ (Cochran, 1954; Higgins y Thompson, 2002). En caso de existir heterogeneidad se realizaron meta-regresiones con la finalidad de explicar el origen de dicha variabilidad (Borenstein y col., 2011).

Se utilizó un modelo de efectos aleatorios según las recomendaciones de Sauvant y col., (2008). Se ejecutaron 9 meta-análisis a partir de los 13 artículos científicos ( $\mathrm{n}=1902$ animales). Las variables analizadas fueron ganancia diaria de peso, consumo diario promedio de alimento y conversión alimentaria. En cada variable se realizaron 3 meta-análisis según la duración de la suplementación de glutamina: de 7 a 30 días, de 7 a 14 días y de 15 a 30 días. Este trabajo de investigación no siguió los protocolos establecidos por PRISMA-P (Moher y col., 2015) pues estos han sido desarrollados para estudios en humanos. Este estudio de meta-análisis sigue una metodología propia de estudios en ciencia animal, como se detalla en varios artículos publicados en los cuales se utilizó meta-análisis en nutrición porcina (Apple y col., 2007; Kiefer y Sanches, 2009; Sales, 2011; Andretta y col., 2012; Létourneau-Montminy y col., 2012; Remus y col., 2015; Hung y col., 2017; MetzlerZebeli y col., 2012; Torres-Pitarch y col., 2017; Zeng, Shurson y Urriola, 2017; Torres-Pitarch y col., 2019).

Tabla 3. Tamaño de efecto de la suplementación de glutamina

\begin{tabular}{|c|c|c|c|c|c|}
\hline \multirow{2}{*}{ Parámetro productivo } & \multirow{2}{*}{ Meta-análisis } & \multicolumn{4}{|c|}{ Tamaño de efecto } \\
\hline & & MD & & & $p$ \\
\hline \multirow{3}{*}{ GDP (kg/día) } & 7 a 30 días & 0,0203 & 0,0070 & 0,0336 & 0,0029 \\
\hline & 7 a 14 días & 0,0146 & $-0,0041$ & 0,0332 & 0,1252 \\
\hline & 15 a 30 días & 0,0282 & 0,0133 & 0,0431 & 0,0002 \\
\hline \multirow{3}{*}{ CDPA ( $k g / d i a)$} & 7 a 30 días & 0,0058 & $-0,0052$ & 0,0168 & 0,3023 \\
\hline & 7 a 14 días & $-0,0033$ & $-0,0128$ & 0,0063 & 0,5030 \\
\hline & 15 a 30 días & 0,0237 & 0,0024 & 0,0450 & 0,0295 \\
\hline \multirow{3}{*}{$\mathrm{CA}(\mathrm{kg} / \mathrm{kg})$} & 7 a 30 días & $-0,0873$ & $-0,1271$ & $-0,0474$ & 0,0000 \\
\hline & 7 a 14 días & $-0,1216$ & $-0,1999$ & $-0,0433$ & 0,0023 \\
\hline & 15 a 30 días & $-0,0706$ & $-0,1120$ & $-0,0290$ & 0,0008 \\
\hline
\end{tabular}

\section{Resultados}

En la Tabla 1 se resumen los principales estadísticos descriptivos calculados (covariables) a partir de los 13 artículos de investigación utilizados. Las variables respuesta GDP, CDPA y CA en cada uno de los periodos evaluados se presentan en la Tabla 2.
El tamaño medio del efecto (expresado en diferencia de medias) de la suplementación de Gln en lechones sobre el rendimiento productivo se presentan en la Tabla 3. Los resultados del índice de inconsistencia, y la prueba de heterogeneidad entre estudios se muestran en la Tabla 4. 
Tabla 4. Índice de inconsistencia

\begin{tabular}{ccc}
\hline Parámetro productivo & Meta-análisis & $I^{2}(\%)$ \\
\hline \multirow{3}{*}{ GDP $(k g / d i ́ a)$} & 7 a 30 días & 81,78 \\
\cline { 2 - 3 } & 7 a 14 días & 87,40 \\
\cline { 2 - 3 } & 15 a 30 días & 36,87 \\
\hline \multirow{2}{*}{ CDPA $(k g / d i ́ a)$} & 7 a 30 días & 48,75 \\
\cline { 2 - 3 } & 7 a 14 días & 20,76 \\
\cline { 2 - 3 } & 15 a 30 días & 45,79 \\
\hline \multirow{2}{*}{$\mathrm{CA}(\mathrm{kg} / \mathrm{kg})$} & 7 a 30 días & 21,20 \\
\cline { 2 - 3 } & 7 a 14 días & 42,33 \\
\cline { 2 - 3 } & 15 a 30 días & 0,00 \\
\hline
\end{tabular}

Los resultados de las meta-regresiones para determinar la influencia de cada una de las covariables sobre las variables productivas de interés en cada periodo se presentan en la Tabla 5 (nivel de inclusión de Gln y duración de suplementación); Tabla 6 (peso vivo y edad al inicio de la suplementación); Tabla 7 (nivel de lisina y proteína cruda en la dieta) y Tabla 7 (número de repeticiones).

\section{Discusión}

La glutamina sirve como una fuente de energía para el crecimiento de los enterocitos y reduce la atrofia yeyunal y el daño en el epitelio intestinal (Wang y col., 2014; Wang y col., 2015). Los lechones que reciben suplementación alimenticia de Gln mejoran la protección de la barrera intestinal, lo cual conduce a una mayor resistencia a los patógenos (Peng y col., 2004), e incremento de la actividad de las enzimas digestivas (Shan y col., 2012), consecuentemente a un mayor aprovechamiento de nutrientes y una mejora en el desempeño productivo (Jiang y col., 2009; Johnson y Lay Jr, 2017). Lo anterior se puede comprobar en el presente trabajo de investigación, pues se encontró que la suplementación alimenticia de Gln mejora $(p<0,001)$ la conversión alimenticia en lechones en cualquiera de los periodos evaluados. Destaca que los lechones que reciben Gln convierten mejor el alimento cuando la suplementación duró entre 7 a 14 días (DM=-0,1216; $p=0,0023)$. Los lechones suplementados requieren $121,6 \mathrm{~g}$ me- nos alimento en comparación con el grupo control para hacer $1 \mathrm{~kg}$ de peso vivo entre 7 a 14 días. Cuando la suplementación se realizó por un periodo más largo (15 a 30 días) el ahorro de alimento es de 70,6 $g$ para hacer un $\mathrm{kg}$ de peso vivo y 87,3 $\mathrm{g}$ cuando la Gln se suplementó entre 7 a 30 días.

Respecto a la ganancia diaria de peso, se observa que la Gln provoca un aumento de ganancia de $20,3 \mathrm{~g} /$ día $(p=0,0029)$ sobre la GDP del grupo control entre los 7 a 30 días de suplementación y de $28,2 \mathrm{~g} /$ día $(p=0,0002)$ entre 15 a 30 días. La variable CDPA únicamente presenta un aumento significativo cuando la administración de Gln fue de 15 a 30 días, consumiéndose 23,7 gramos al día adicionales en comparación con el grupo testigo. De manera similar en pollos de engorde, se encontró que la suplementación alimenticia de glutamina permite un uso más eficiente de los nutrientes ingeridos y por lo tanto una mejora en el rendimiento productivo (Namroud y col., 2017), asociado al efecto de la Gln sobre la longitud de las vellosidades intestinales (Abdulkarimi, Shahir y Daneshyar, 2019). Así se requerirá menor cantidad de alimento para llevar a los cerdos a alcanzar el peso de mercado o se reducirían los días necesarios para llegar al peso de mercado, representando un ahorro importante para la industria porcina. Además, se ha comprobado que la suplementación de Gln favorece el sistema inmunológico de los lechones, garantizando un mejor estado de salud y preservando de esta manera los nutrientes para el crecimiento (Johnson y col., 2006; Zhong y col., 2012). 
Tabla 5. Meta-regresión para nivel de inclusión de Gln (\%) y duración de suplementación (días)

\begin{tabular}{|c|c|c|c|c|c|c|c|c|c|}
\hline \multirow{3}{*}{$\begin{array}{l}\text { Parámetro } \\
\text { productivo }\end{array}$} & \multirow{3}{*}{$\begin{array}{l}\text { Meta- } \\
\text { regresión }\end{array}$} & \multicolumn{4}{|c|}{ Nivel de inclusión de Gln (\%) } & \multicolumn{4}{|c|}{ Duración de suplementación (días) } \\
\hline & & \multicolumn{2}{|c|}{ Intercepto } & \multicolumn{2}{|c|}{ Coef. Regresión } & \multicolumn{2}{|c|}{ Intercepto } & \multicolumn{2}{|c|}{ Coef. Regresión } \\
\hline & & Estimado & $p$ & Estimado & $p$ & Estimado & $p$ & Estimado & $p$ \\
\hline \multirow{3}{*}{$\begin{array}{c}\text { GDP } \\
(\mathrm{kg} / d i a)\end{array}$} & $7 \mathrm{a} 3$ & 0,024 & 0,007 & 0,006 & 0,503 & 0,041 & $<0,001$ & $-0,001$ & 0,002 \\
\hline & 7 a 14 días & 0,025 & 0,010 & 0,005 & 0,578 & 0,094 & $<0,001$ & $-0,007$ & $<0,001$ \\
\hline & 15 a 30 días & 0,014 & 0,505 & 0,011 & 0,577 & 0,121 & 0,002 & $-0,004$ & 0,013 \\
\hline \multirow{3}{*}{$\begin{array}{l}\text { CDPA } \\
\text { (kg/día) }\end{array}$} & 7 a 30 días & 0,003 & 0,762 & $-0,003$ & 0,737 & $-0,019$ & 0,002 & 0,002 & $<0,001$ \\
\hline & 7 a 14 días & 0,004 & 0,700 & $-0,010$ & 0,372 & $-0,025$ & 0,064 & 0,002 & 0,117 \\
\hline & 15 a 30 días & 0,048 & 0,176 & $-0,025$ & 0,475 & 0,097 & 0,018 & $-0,003$ & 0,069 \\
\hline \multirow{3}{*}{$\begin{array}{c}\text { CA } \\
(\mathrm{kg} / \mathrm{kg})\end{array}$} & 7 a 30 días & $-0,050$ & 0,044 & $-0,021$ & 0,434 & $-0,063$ & 0,143 & $-0,0002$ & 0,927 \\
\hline & 7 a 14 días & $-0,050$ & 0,051 & $-0,025$ & 0,456 & $-0,260$ & 0,260 & 0,015 & 0,390 \\
\hline & 15 a 30 días & $-0,054$ & 0,599 & $-0,016$ & 0,873 & $-0,080$ & 0,638 & 0,0004 & 0,953 \\
\hline
\end{tabular}

$p=$ valor de probabilidad

Tabla 6. Meta-regresión para peso vivo (kg) y edad (días) al inicio de la suplementación de Gln

\begin{tabular}{|c|c|c|c|c|c|c|c|c|c|}
\hline \multirow{3}{*}{$\begin{array}{l}\text { Parámetro } \\
\text { productivo }\end{array}$} & \multirow{3}{*}{$\begin{array}{l}\text { Meta- } \\
\text { regresión }\end{array}$} & \multicolumn{4}{|c|}{ Peso vivo inicio de suplementación (kg) } & \multicolumn{4}{|c|}{ Edad inicio de suplementación (días) } \\
\hline & & \multicolumn{2}{|c|}{ Intercepto } & \multicolumn{2}{|c|}{ Coef. Regresión } & \multicolumn{2}{|c|}{ Intercepto } & \multicolumn{2}{|c|}{ Coef. Regresión } \\
\hline & & Estimado & $p$ & Estimado & $p$ & Estimado & $p$ & Estimado & $p$ \\
\hline \multirow{3}{*}{$\begin{array}{l}\text { GDP } \\
\left(\mathrm{kg} / d i a^{2}\right)\end{array}$} & 7 a 30 días & $-0,086$ & $<0,001$ & 0,019 & $<0,001$ & 0,043 & 0,041 & $-0,001$ & 0,528 \\
\hline & 7 a 12 & $-0,138$ & $<0,001$ & 0, & $<0,001$ & 0,020 & 0 , & 0,001 & 0,797 \\
\hline & 15 a 30 días & 0,027 & 0,428 & 0,000 & 0,979 & 0,047 & 29 & $-0,001$ & 0,589 \\
\hline \multirow{3}{*}{$\begin{array}{l}\text { CDPA } \\
\text { (kg/día) }\end{array}$} & 7 a 30 días & $-0,020$ & 0,118 & 0,004 & 0,114 & $-0,020$ & 0,324 & 0,001 & 0,343 \\
\hline & 7 a 14 días & $-0,015$ & 0,286 & 0,002 & 0,449 & $-0,014$ & 0,586 & 0,000 & 0,716 \\
\hline & 15 a 30 días & 0,030 & 0,399 & $-0,001$ & 0,839 & 0,072 & 0,119 & $-0,002$ & 0,284 \\
\hline \multirow{3}{*}{$\begin{array}{c}\mathrm{CA} \\
(\mathrm{kg} / \mathrm{kg})\end{array}$} & 7 a 30 días & 0,124 & 0,169 & $-0,030$ & 0,032 & 0,066 & 0,386 & $-0,006$ & 0,074 \\
\hline & 7 a 14 días & 0,227 & 0,103 & $-0,050$ & 0,035 & 0,415 & 0,005 & $-0,025$ & 0,001 \\
\hline & 15 a 30 días & 0,133 & 0,415 & $-0,029$ & 0,209 & $-0,026$ & 0,860 & $-0,002$ & 0,767 \\
\hline
\end{tabular}

$p=$ valor de probabilidad

Respecto a la heterogeneidad entre estudios, se reporta que únicamente en la GDP (7 a 30d y 7 a $14 d)$ la variabilidad es alta $(>75 \%)$. En las demás variables la heterogeneidad se encuentra entre baja a moderada $(<50 \%)$. Con la finalidad de explicar la variabilidad existente entre estudios se realizaron meta-regresiones entre la variable respuesta y cada una de las co-variables señaladas anteriormente. El nivel de inclusión de Gln (\%) en la dieta suplementada a los lechones no tiene impacto $(p>0,05)$ sobre ninguna de las variables productivas evaluadas. El nivel más utilizado es de $1 \%$, sin embargo, los va-

La duración de la suplementación (días) influye en forma significativa $(p<0,05)$ sobre la GDP (todos los periodos evaluados) y CDPA (7 a 30 días). El peso vivo del lechón con el cual inicia el periodo de suplementación también tiene un efecto signifi- lores oscilan entre 0,1 a $2 \%$. En estudios realizados en pollos se reporta que la suplementación de niveles altos de glutamina afecta de manera negativa el desempeño productivo, debido a que causan una reducción del consumo de alimento, como se observa en los resultados encontrados cuando se utilizó un nivel de $4 \%$. Adicionalmente, los niveles altos de Gln generan un desbalance con los demás aminoácidos de la dieta, afectando su absorción intestinal a pesar de evidenciar un aumento en el tamaño de las vellosidades intestinales (Bartell y Batal, 2007).

cativo $(p<0,05)$ sobre la GDP y CA en los periodos de 7 a 30 días y de 7 a 14 días. Se aprecia que a medida que aumenta el peso vivo del lechón aumenta la GDP y disminuye la CA. Los valores del peso vivo oscilan entre 4 a $16 \mathrm{~kg}$ y una media de 
Tabla 7. Meta-regresión para nivel de lisina (\%) y proteína cruda en la dieta (\%)

\begin{tabular}{|c|c|c|c|c|c|c|c|c|c|}
\hline \multirow{3}{*}{$\begin{array}{l}\text { Parámetro } \\
\text { productivo }\end{array}$} & \multirow{3}{*}{$\begin{array}{l}\text { Meta- } \\
\text { regresión }\end{array}$} & \multicolumn{4}{|c|}{ Nivel de lisina en la dieta (\%) } & \multicolumn{4}{|c|}{ Nivel de PC en la dieta (\%) } \\
\hline & & \multicolumn{2}{|c|}{ Intercepto } & \multicolumn{2}{|c|}{ Coef. Regresión } & \multicolumn{2}{|c|}{ Intercepto } & \multicolumn{2}{|c|}{ Coef. Regresión } \\
\hline & & Estimado & $p$ & Estimado & $p$ & Estimado & $p$ & Estimado & $p$ \\
\hline \multirow{3}{*}{$\begin{array}{c}\text { GDP } \\
(\mathrm{kg} / \text { día })\end{array}$} & 7 a 30 días & $-0,145$ & $<0,001$ & 0,126 & $<0,001$ & $-0,152$ & $<0,001$ & 0,009 & $<0,001$ \\
\hline & 7 a 14 días & $-0,165$ & $<0,001$ & 0,140 & $<0,001$ & $-0,215$ & $<0,001$ & 0,012 & $<0,001$ \\
\hline & 15 a 30 días & 0,185 & 0,019 & $-0,118$ & 0,043 & 0,192 & 0,015 & $-0,008$ & 0,036 \\
\hline \multirow{3}{*}{$\begin{array}{l}\text { CDPA } \\
\text { (kg/día) }\end{array}$} & 7 a 30 días & $-0,041$ & 0,335 & 0,033 & 0,337 & $-0,022$ & 0,59 & 0,001 & 0,593 \\
\hline & 7 a 14 días & $-0,028$ & 0,591 & 0,019 & 0,654 & $-0,021$ & 0,643 & 0,001 & 0,715 \\
\hline & 15 a 30 días & 0,183 & 0,051 & $-0,121$ & 0,089 & 0,293 & 0,008 & $-0,013$ & 0,014 \\
\hline \multirow{3}{*}{$\begin{array}{c}\mathrm{CA} \\
(\mathrm{kg} / \mathrm{kg})\end{array}$} & 7 a 30 días & $-0,137$ & 0,495 & 0,052 & 0,725 & $-0,716$ & 0,002 & 0,029 & 0,004 \\
\hline & 7 a 14 días & $-0,162$ & 0,482 & 0,075 & 0,669 & $-1,370$ & $<0,001$ & 0,057 & $<0,001$ \\
\hline & 15 a 30 días & $-0,219$ & 0,721 & 0,106 & 0,809 & $-0,285$ & 0,430 & 0,010 & 0,552 \\
\hline
\end{tabular}

$p=$ valor de probabilidad

$\mathrm{PC}=$ proteína cruda

Tabla 8. Meta-regresión para número de repeticiones

\begin{tabular}{|c|c|c|c|c|c|}
\hline \multirow{3}{*}{$\begin{array}{l}\text { Parámetro } \\
\text { productivo }\end{array}$} & \multirow{3}{*}{$\begin{array}{l}\text { Meta- } \\
\text { regresión }\end{array}$} & \multicolumn{4}{|c|}{ Número de repeticiones } \\
\hline & & \multicolumn{2}{|c|}{ Intercepto } & \multicolumn{2}{|c|}{ Coef. Regresión } \\
\hline & & Estimado & $p$ & Estimado & $p$ \\
\hline \multirow{3}{*}{ GDP (kg/día) } & 7 a 30 días & $-0,006$ & 0,143 & 0,005 & $<0,001$ \\
\hline & 7 a 14 días & $-0,010$ & 0,022 & 0,006 & $<0,001$ \\
\hline & 15 a 30 días & 0,030 & 0,071 & $-0,001$ & 0,819 \\
\hline \multirow{3}{*}{ CDPA ( $k g / d i ́ a)$} & 7 a 30 días & 0,008 & 0,209 & $-0,002$ & 0,153 \\
\hline & 7 a 14 días & 0,006 & 0,393 & $-0,003$ & 0,079 \\
\hline & 15 a 30 días & 0,064 & $<0,001$ & $-0,009$ & 0,024 \\
\hline \multirow{3}{*}{$\mathrm{CA}(\mathrm{kg} / \mathrm{kg})$} & 7 a 30 días & $-0,036$ & 0,211 & $-0,007$ & 0,245 \\
\hline & 7 a 14 días & $-0,032$ & 0,313 & $-0,009$ & 0,199 \\
\hline & 15 a 30 días & $-0,070$ & 0,384 & 0,001 & 0,972 \\
\hline
\end{tabular}

$6,89 \mathrm{~kg}$. La edad del lechón a la cual se inicia la suplementación únicamente tiene influencia en la CA cuando la suplementación duró entre 7 a 14 días. De los estudios utilizados se encontró que el rango de la edad de inicio de la suplementación de Gln está entre 17 a 28 días, siendo 21 días la edad con mayor frecuencia. Es importante considerar que la Gln natural constituye apenas un $10 \%$ del contenido de aminoácidos de la proteína total en una dieta convencional para cerdos, siendo necesaria la suplementación para alcanzar un máximo crecimiento y facilitar el normal funcionamiento intestinal, particularmente en estados hipercatabólicos (Wu, 2014) que ocurren durante el destete de los lechones debido al estrés social y ambiental (Spreeuwenberg y col., 2001).

Algunos factores relacionados con la formula- ción de la dieta también tienen efecto significativo sobre la GDP, CDPA y CA tras la suplementación de Gln en lechones. El nivel de lisina $(p<0,05)$ y proteína cruda $(p<0,05)$ afectan la GDP en los 3 periodos evaluados. Se evidencia que a medida que el nivel de lisina y PC aumenta la GDP también aumenta para los periodos 7 a 30 días y 7 a 14 días. Sin embargo, en el periodo 15 a 30 días hay una reducción de la GDP. Adicionalmente, el nivel de PC afectó la CDPA cuando la suplementación duró entre 15 a 30 días $(p=0,014)$ y la CA cuando la administración de Gln fue de 7 a 30 días $(p=0,004)$ y 7 a 14 días $(p<0,001)$. En este trabajo los valores de PC y lisina de las dietas utilizadas en lechones estuvieron entre 18 a $23 \%$ y 1,17 a 1,6\%, respectivamente. Una estrategia para mejorar la utilización de proteína en lechones y prevenir desórdenes intestinales es la reducción del nivel de proteína cruda 
concomitantemente con una adecuada suplementación de aminoácidos libres (sintéticos) (Le Floc'h y col., 2018). Gloaguen y col., (2014) confirma la eficacia de esta estrategia y la posibilidad de formular dietas con bajos niveles de PC $(13,5 \%)$ para lechones entre 10 a $20 \mathrm{~kg}$ de peso vivo. Adicionalmente, las dietas con restricción moderada de proteína (13$15,3 \%$ ) demostraron ser beneficiosas para la salud de la microbiota intestinal, la actividad metabólica en el intestino grueso y la mejora en la función de la barrera intestinal de lechones (Peng y col., 2017). La reducción del contenido de PC permite reducir el consumo de nitrógeno y evita el exceso de aminoácidos, por lo tanto, previene sobrecargas metabólicas.

Finalmente se determinó que el número de repeticiones influye sobre la GDP para los periodos 7 a $30(p<0,001)$ y 7 a 14 días $(p<0,001)$, así como en el CDPA cuando la suplementación duró entre 15 a 30 días $(p=0,024)$. El número de repeticiones en los distintos trabajos utilizados para este estudio van desde 2 hasta 12, siendo 3 el más utilizado. Lo anterior confirma la importancia señalada por Aaron y Hays, (2004) de considerar un número apropiado de individuos para ser usados en estudios experimentales en cerdos, pues ante un número inadecuado de repeticiones por tratamiento es posible que no se lleguen a detectar diferencias significativas, generando pérdida de tiempo y dinero para el investigador.

\section{Conclusión}

La suplementación de glutamina mejora el desempeño productivo en lechones, transformándose en una opción firme para ser utilizada en dietas de destete para disminuir las pérdidas generadas por el estrés durante esta etapa.

\section{Referencias}

Aaron, D. K. y V. W. Hays (2004). «How many pigs? Statistical power considerations in swine nutrition experiments». En: Journal of animal science 82.13, E245-E254. Onine: https : / / bit.ly / 2GxHTxP.
Abdulkarimi, R., M. H. Shahir y M. Daneshyar (2019). «Effects of dietary glutamine and arginine supplementation on performance, intestinal morphology and ascites mortality in broiler chickens reared under cold environment». En: Asian-Australasian Journal of Animal Sciences 32.1, 110-117. Online: https:/ / bit.ly/38e5UWF.

Abreu, M. y col. (2010). «Glutamina, nucleotídeos e plasma suíno em raogões para leitões desmamados». En: R. Bras. Zootec 39.3, 520-525. Online: https:/ / bit.ly /2IyrJX9.

Andretta, I. y col. (2012). «Meta-analysis of the relationship between ractopamine and dietary lysine levels on carcass characteristics in pigs». En: Livestock Science 143.1, 91-96. Online: https:/ / bit. ly/2Ls5sKy.

Apple, J. K. y col. (2007). «Meta-analysis of the ractopamine response in finishing swine». En: The Professional Animal Scientist 23.3, 179-196. Online: https://bit.ly/2OVJkdF.

Bartell, S. M. y A. B. Batal (2007). «The effect of supplemental glutamine on growth performance, development of the gastrointestinal tract, and humoral immune response of broilers». En: Poultry Science 86.9, 1940-1947. Online: https: / / bit.ly/2T39mN0.

Bax, L. (2016). MIX 2.0 -Professional software for meta-analysis in Excel. Version 2.0. BiostatXL. URL: Online: \url\{https://bit.ly/2VckrhX\}.

Borenstein, M. y col. (2011). Introduction to metaanalysis. Chichester: John Wiley \& Sons, Online: https://bit.ly/2ZoqhvV.

Boza, J. J. y col. (2000). «Role of glutamine on the de novo purine nucleotide synthesis in Caco2 cells». En: European journal of nutrition 39.1, 38-46. Online: https://bit.ly/2Iyrs6W.

Campbell, J. M., J. D. Crenshaw y J. Polo (2013). «The biological stress of early weaned piglets». En: Journal of animal science and biotechnology 4.19, Online: https://bit.ly/2Xt1EfN.

Chamorro, S. y col. (2010). «Effect of dietary supplementation with glutamine and a combination of glutamine-arginine on intestinal health in twenty-five-day-old weaned rabbits». En: Journal of animal science 88.1, 170-180. Online: https: //bit.ly/2Pjt5px.

Cochran, W. G. (1954). «The combination of estimates from different experiments». En: Biometrics 10.1, 101-129. Online:https:/ / bit.ly/2UO6QOc.

Cromwell, G. L. (2002). «Why and how antibiotics are used in swine production». En: Animal bio- 
technology 13.1, 7-27. Online: https: / / bit.ly / 2UKuX0q.

Curi, R. y col. (2007). «Glutamine, gene expression, and cell function». En: Front Biosci 12.1, 344-357. Online: https:/ / bit.ly/2VZD4mu.

Domeneghini, C. y col. (2004). «Structural patterns of swine ileal mucosa following L-glutamine and nucleotide administration during the weaning period. An histochemical and histometrical study». En: Histology and Histopathology 19.1, 49-58. Online: https:/ / bit.ly/2GxXIEY.

Duttlinger, A. W. y col. (2019). «Replacing dietary antibiotics with 0.20 nursery diets: Impact on health and productivity of pigs following weaning and transport». En: Journal of Animal Science 97, 2035-2052. Online: https:/ / bit.ly/2VXZTa3.

Gloaguen, M. y col. (2014). «The use of free amino acids allows formulating very low crude protein diets for piglets». En: Journal of animal science 92.2, 637-644. Online: https://bit.ly/2s0Wzkk.

Gresse, R. y col. (2017). «Gut microbiota dysbiosis in postweaning piglets: understanding the keys to health». En: Trends in microbiology 25.10, 851-873. Online: https:/ / bit.ly/2ISCqDF.

Haynes, T. E. y col. (2009). «L-Glutamine or L-alanyl-L-glutamine prevents oxidant-or endotoxin-induced death of neonatal enterocytes». En: Amino acids 37.1, 131-142. Online: https: / / bit.ly/2GnZXJI.

He, J. y col. (2016). «Effects of L-glutamine on growth performance, antioxidant ability, immunity and expression of genes related to intestinal health in weanling pigs». En: Livestock Science 189, 102-109. Online: https:/ / bit.ly /2Uv7ScU.

Higgins, J. P. y S. G. Thompson (2002). «Quantifying heterogeneity in a metaanalysis». En: Statistics in medicine 21.11, 1539-1558. Online: https: / / bit.ly/2UMynQq.

Horio, Y. y col. (2008). «Glutamine supplementation increases Th1-cytokine responses in murine intestinal intraepithelial lymphocytes». En: Cytokine 44.1, 92-95. Online: https: / / bit.ly/2Vh7G5C.

Hsu, C. B. y col. (2010). "The effect of glutamine supplement on small intestinal morphology and xylose absorptive ability of weaned piglets». En: African Journal of Biotechnology 9.41, 7003-7008. Online: https:/ / bit.ly/2PhX5Ss.

Hung, Y. T. y col. (2017). «Peroxidized lipids reduce growth performance of poultry and swine: a meta-analysis». En: Animal Feed Science and
Technology 231, 47-58. Online: https: / / bit.ly / 2rZU81q.

Jazideh, F. y col. (2014). «The effects of dietary glutamine supplementation on growth performance and intestinal morphology of broiler chickens reared under hot conditions». En: Turkish Journal of Veterinary and Animal Sciences 38.3, 264-270. Online: https:/ / bit.ly/2UuAUJT.

Jiang, Z. Y. y col. (2009). «Effects of dietary glycylglutamine on growth performance, small intestinal integrity, and immune responses of weaning piglets challenged with lipopolysaccharide». En: Journal of Animal Science 87.12, 4050-4056. Online: https://bit.ly/2VWQhMB.

Johnson, I. R. y col. (2006). «Glutamine supplementation influences immune development in the newly weaned piglet». En: Developmental $\mathcal{E}$ Comparative Immunology 30.12, 1191-1202. Online: https:/ / bit.ly/2reFe7E.

Johnson, J. S. y D. C. Lay Jr (2017). «Evaluating the behavior, growth performance, immune parameters, and intestinal morphology of weaned piglets after simulated transport and heat stress when antibiotics are eliminated from the diet or replaced with L-glutamine». En: Journal of animal science 95.1, 91-102. Online: https: / / bit.ly / 2XC9AeT.

Kiefer, C. y J. F. Sanches (2009). «Metanálise dos níveis de ractopamina em dietas para suínos em terminação». En: Revista Brasileira de Zootecnia 38.6, 1037-1044. Online: https:/ / bit.ly/2rjTADu.

Lalles, J. P. y col. (2007). «Nutritional management of gut health in pigs around weaning». En: Proceedings of the Nutrition Society 66.2, 260-268. Online: https:/ / bit.ly/2XvJTMY.

Le Floc'h, N. y col. (2018). «The relevance of functional amino acids to support the health of growing pigs». En: Animal Feed Science and Technology 245, 104-116. Online: https:/ / bit.ly/2Ppr7nO.

Lee, D. N. y col. (2003). «Effect of dietary glutamine supplement on performance and intestinal morphology of weaned pigs». En: Asian-australasian journal of animal sciences 16.12, 1770-1776. Online: https:/ / bit.ly/2XsS50f.

Létourneau-Montminy, M. P. y col. (2012). «Metaanalysis of phosphorus utilization by growing pigs: effect of dietary phosphorus, calcium and exogenous phytase». En: Animal 6.10, 1590-1600. Online: https://bit.ly/38i4BpR.

Luquetti, B. C. y col. (2016). «Effects of glutamine on performance and intestinal mucosa morpho-

La Granja: Revista de Ciencias de la Vida 31(1) 2020:96-107.

(C)2020, Universidad Politécnica Salesiana, Ecuador. 
metry of broiler chickens vaccinated against coccidiosis». En: Scientia Agricola 73.4, 322-327. Online: https:/ / bit.ly/2VdafWw.

Maiorka, A. y col. (2016). «Effect of broiler breeder age and glutamine supplementation on the development of the intestinal mucosa of 7-day-old chicks». En: Revista Brasileira de Ciência Avícola 18.1, 17-22. Online: https:/ /bit.ly/2VXMA9o.

Manvailer, G. V. y col. (2015). «Glutamine for broilers reared in hot environment». En: Archivos de Zootecnia 64.248, 377-382. Online: https: / / bit . ly/2HOv4PG.

Metzler-Zebeli, B. U. y col. (2012). «Assessing the effect of dietary inulin supplementation on gastrointestinal fermentation, digestibility and growth in pigs: A meta-analysis». En: Animal Feed Science and Technology 233, 120-132. Online: https: / / bit.ly/2sShs1E.

Moeser, A. J., C. S. Pohl y M. Rajput (2017). «Weaning stress and gastrointestinal barrier development: Implications for lifelong gut health in pigs». En: Animal Nutrition 3.4, 313-321. Online: https://bit.ly/2UMQgP3.

Moher, D. y col. (2015). «Preferred reporting items for systematic review and meta-analysis protocols (PRISMA-P) 2015 statement». En: Systematic reviews 4.1, 1-8. Online: https:/ / bit.ly/2sN1Lso.

Muro, E. M. y col. (2015). «Aditivos fitogênicos e glutamina mais ácido glutâmico na dieta de frangos desafiados com coccidiose». En: Agrarian 8.29, 304-311. Online: https : / / bit . ly / $2 \mathrm{KSJVwV}$.

Namroud, N. F. y col. (2017). «Impact of dietary glutamine on amino acid digestibility values and intestinal morphometric parameters in neonate chicks». En: South African Journal of Animal Science 47.4, 440-453. Online: https: / / bit.ly / 2XBa5G3.

Nascimento, G. M. y col. (2014). «Performance and intestinal characteristics of broiler chicken fed diet with glutamine in the diet without anticoccidials agents». En: Revista Brasileira de Saúde e Produção Animal 15.3, 637-648. Online: https: / / bit.ly/2UuBg39.

Nassiri Moghaddam, H. y A. H. AlizadehGhamsari (2013). «Improved performance and small intestinal development of broiler chickens by dietary L-glutamine supplementation». En: Journal of applied animal research 41.1, 1-7. Online: https://bit.ly/2PqveQk.
Olubodun, J. O. y col. (2015). «Glutamine and glutamic acid supplementation enhances performance of broiler chickens under the hot and humid tropical condition». En: Italian Journal of Animal Science 14.1, 3263. Online: https: / / bit.ly / 2IyMYIo.

Peng, X. y col. (2004). «Effects of enteral supplementation with glutamine granules on intestinal mucosal barrier function in severe burned patients». En: Burns 30.2, 135-139. Online: https: //bit.ly/2zPWRf9.

Peng, Y. y col. (2017). «Progressive response of large intestinal bacterial community and fermentation to the stepwise decrease of dietary crude protein level in growing pigs». En: Applied microbiology and biotechnology 101.13, 5415-5426. Online: https://bit.ly/34ZDZaO.

Pluske, J. R., D. L. Turpin y J. C. Kim (2018). «Gastrointestinal tract (gut) health in the young pig». En: Animal Nutrition 4.2, 187-196. Online: https: //bit.ly/2XBHUXv.

Remus, A. y col. (2015). «Exploratory study on the utilization of different dietary methionine sources and methionine to lysine ratio for growing-finishing pigs». En: Livestock Science 181, 96-102. Online: https:/ / bit.ly/2YpVmPu.

Rhoads, J. M. y G. Wu (2009). "Glutamine, arginine, and leucine signaling in the intestine». En: Amino acids 37.1, 111-122. Online: https: / / bit . ly/2Iwvexo.

Ribeiro Jr, V. y col. (2015). «Effects of Dietary L-Glutamine or L-Glutamine Plus L-Glutamic Acid Supplementation Programs on the Performance and Breast Meat Yield Uniformity of 42d-Old Broilers». En: Brazilian Journal of Poultry Science 17.SPE, 93-98. Online: https: / / bit.ly / 2Gp1yPi.

Sakiyama, T. y col. (2009). «Glutamine increases autophagy under basal and stressed conditions in intestinal epithelial cells». En: Gastroenterology 136.3, 924-932. Online: https:/ / bit.ly /2IGV7Kl.

Sales, J. (2011). "A meta-analysis of the effects of dietary betaine supplementation on finishing performance and carcass characteristics of pigs». En: Animal Feed Science and technology 165.1-2, 68-78. Online: https:/ /bit.ly/2YmDGnW.

Sauvant, D. y col. (2008). «Meta-analyses of experimental data in animal nutrition». En: Animal 2.8, 1203-1214. Online: https://bit.ly/39Vgq5a.

Shan, Y. y col. (2012). «Dietary supplementation of arginine and glutamine enhances the growth 
and intestinal mucosa development of weaned piglets». En: Livestock Science 150.1-3, 369-373. Online: https:/ / bit.ly/2UJy4Wi.

Smith, M. G. y col. (2010). «Antimicrobial resistance and virulence gene profiles in multi-drug resistant enterotoxigenic Escherichia coli isolated from pigs with post-weaning diarrhoea». En: Veterinary microbiology 145.3-4, 299-307. Online: https://bit.ly/2IHZyEu.

Solà-Oriol, D. y J. Gasa (2017). «Feeding strategies in pig production: Sows and their piglets». En: Animal feed Science and technology 233, 34-52. Online: https:/ / bit.ly/2GB3A0d.

Spreeuwenberg, M. A. M. y col. (2001). «Small intestine epithelial barrier function is compromised in pigs with low feed intake at weaning». En: The Journal of nutrition 131.5, 1520-1527. Online: https://bit.ly /2s0AX7G.

Torres-Pitarch, A. y col. (2017). «Effect of feed enzymes on digestibility and growth in weaned pigs: A systematic review and meta-analysis». En: Animal Feed Science and Technology 233, 145-159. Online: https:/ / bit.ly/2DPa5KF.

Torres-Pitarch, A. y col. (2019). «Systematic review and meta-analysis of the effect of feed enzymes on growth and nutrient digestibility in growfinisher pigs: Effect of enzyme type and cereal source». En: Animal Feed Science and Technology 251, 153-165. Online: https:/ / bit.ly /2rbVRAU.

Varley, M. A. y J. Wiseman (2001). The weaner pig: nutrition and management. New York: CABI, Online: https:/ / bit.ly/2UuCwmV.

Wang, B. y col. (2015). «Glutamine and intestinal barrier function». En: Amino acids 47.10, 2143-2154. Online: https:/ / bit.ly/2VhbEeG.

Wang, H. y col. (2014). «Glutamine enhances tight junction protein expression and modulates corticotropin-releasing factor signaling in the jejunum of weanling piglets». En: The Journal of nutrition 145.1, 25-31. Online: https: / / bit.ly / 2Gyy5DO.

Watford, M. (2015). «Glutamine and glutamate: Nonessential or essential amino acids?» En: Animal Nutrition 1.3, 119-122. Online: https: / / bit.ly / 2GpLxsA.

Wijtten, P. J., J. van der Meulen y M. W. Verstegen (2011). «Intestinal barrier function and absorption in pigs after weaning: a review». En: British Journal of Nutrition 105.7, 967-981. Online: https:/ / bit.ly/2KVfhTq.

Wu, G. (2014). «Dietary requirements of synthesizable amino acids by animals: a paradigm shift in protein nutrition». En: Journal of Animal Science and Biotechnology 5.1, 34. Online: https://bit.ly/ 2OUFBgz.

Wu, G., S. A. Meier y D. A. Knabe (1996). «Dietary glutamine supplementation prevents jejunal atrophy in weaned pigs». En: The Journal of nutrition 126.10, 2578-2584. Online: https: / / bit. ly/2wCPKHX.

Wu, G. y col. (2011). «Triennial Growth Symposium: important roles for L-glutamine in swine nutrition and production». En: Journal of Animal Science 89.7, 2017-2030. Online: https : / / bit . ly / 2IxO8UN.

Xi, P. y col. (2011). «Regulation of protein metabolism by glutamine: implications for nutrition and health». En: Front Biosci 16.1, 578-597. Online: https://bit.ly/2Gqsled.

Xiao, Y. P. y col. (2012). «Response to dietary Lglutamine supplementation in weaned piglets: a serum metabolomic comparison and hepatic metabolic regulation analysis». En: Journal of animal science 90.12, 4421-4430. Online: https:/ / bit. ly/2Gr0rPf.

Zeng, Z. K., G. C. Shurson y P. E. Urriola (2017). «Prediction of the concentration of standardized ileal digestible amino acids and safety margins among sources of distillers dried grains with solubles for growing pigs: A meta-analysis approach». En: Animal feed science and technology 231, 150-159. Online: https://bit.ly/2sQ5vcF.

Zhong, X. y col. (2011). "Intestinal growth and morphology is associated with the increase in heat shock protein 70 expression in weaning piglets through supplementation with glutamine». En: Journal of animal science 89.11, 3634-3642. Online: https:/ / bit.ly/2XCf3lV.

Zhong, X. y col. (2012). «Effects of glutamine supplementation on the immune status in weaning piglets with intrauterine growth retardation». En: Archives of animal nutrition 66.5, 347-356. Online: https:/ / bit.ly/2Iz0Z9d.

Zhou, R. Y. y col. (2006). "Effects of biocom as a replacement of glutamine on performance and blood biochemical indexes of early weaned piglets». En: Asian-australasian journal of animal sciences 19.6, 872-876. Online: https: / / bit.ly / 2DpwbU6.

Zou, X. T. y col. (2006). «Effects of glutamine on growth performance of weanling piglets». En: Czech Journal of Animal Science 51.10, 444-448. Online: https:/ / bit.ly/2vkm3Y9. 